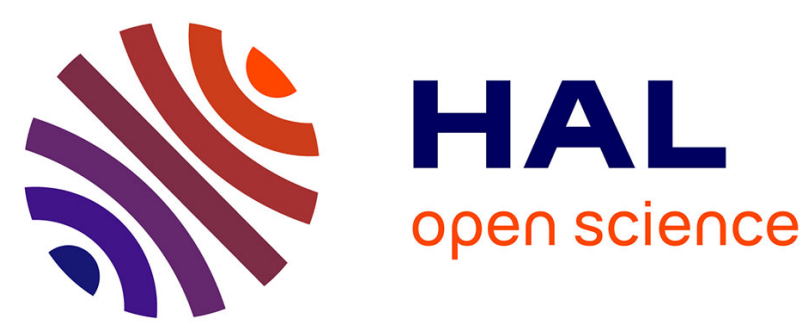

\title{
COMPARISON OF THREE LIGHT DOSES IN THE PHOTODYNAMIC TREATMENT OF ACTINIC KERATOSIS USING MATHEMATICAL MODELING
}

Anne-Sophie Vignion-Dewalle, Nacim Betrouni, Maximilien Vermandel, Laurent Mortier, Serge Mordon

\section{To cite this version:}

Anne-Sophie Vignion-Dewalle, Nacim Betrouni, Maximilien Vermandel, Laurent Mortier, Serge Mordon. COMPARISON OF THREE LIGHT DOSES IN THE PHOTODYNAMIC TREATMENT OF ACTINIC KERATOSIS USING MATHEMATICAL MODELING . Journal of Biomedical Optics, 2015, pp.058001. hal-01167978

\section{HAL Id: hal-01167978 \\ https://hal.science/hal-01167978}

Submitted on 7 Sep 2015

HAL is a multi-disciplinary open access archive for the deposit and dissemination of scientific research documents, whether they are published or not. The documents may come from teaching and research institutions in France or abroad, or from public or private research centers.
L'archive ouverte pluridisciplinaire HAL, est destinée au dépôt et à la diffusion de documents scientifiques de niveau recherche, publiés ou non, émanant des établissements d'enseignement et de recherche français ou étrangers, des laboratoires publics ou privés. 


\title{
Comparison of three light doses in the photodynamic treatment of actinic keratosis using mathematical modeling
}

\author{
Anne-Sophie Vignion-Dewalle ${ }^{1}$, Nacim Betrouni ${ }^{1,2}$, Maximilien Vermandel ${ }^{1,2,3}$, Laurent \\ Mortier $^{1,2,3}$, Serge Mordon ${ }^{1,2}$ \\ ${ }^{1}$ INSERM U703, F-59000 Lille, France \\ ${ }^{2}$ Univ Lille Nord de France, F-59000 Lille, France \\ ${ }^{3} \mathrm{CHU}$ Lille, F-59000 Lille, France
}

\begin{abstract}
-
Purpose: Photodynamic therapy is an emerging treatment modality for various diseases, especially for cancer therapy. Although high efficacy is demonstrated for photodynamic therapy using standardized protocolsin non-hyperkeratotic actinic keratoses, alternative light dosesexpected to increase efficiency, to reduce adverse effects or to expand the use of photodynamic therapy, are stillbeing evaluated and refined. In this paper, we propose a comparison of the three most common light doses in the treatment of actinic keratosis with 5aminolevulinic acid photodynamic therapy through mathematical modeling.
\end{abstract}

Methods: The proposed modelis based on an iterative procedure that involves determination of the local fluence rate, updating of the local optical properties and estimation of the localdamage induced by the therapy. This model was applied,on a simplified skin sample model including an actinic keratosis lesion,with three different light doses (red light dose, 37 $\mathrm{J} / \mathrm{cm}^{2}, 75 \mathrm{~mW} / \mathrm{cm}^{2}, 500 \mathrm{~s}$; blue light dose, $10 \mathrm{~J} / \mathrm{cm}^{2}, 10 \mathrm{~mW} / \mathrm{cm}^{2}, 1000 \mathrm{~s}$; daylight dose, 9000 s) reported in the literature.The reference damage,defined as the minimum of the three local damagesobtained at the deepest part of the actinic keratosis, was first determined. The treatment times required with the fluence rates of the three common light doses to achieve this reference damage to the deepest part of the actinic keratosiswere then estimated and compared.

Results:Determined almost four and three times lower than the local damagesobtained by the blue light doseand the daylight dose respectively, the local damage obtained by the red light dose was foundto be thereferencedamage.This reference damage was achieved at the deepest 
part in the AK by the above-mentioned blue light fluence rate and daylight fluence rate using treatment timesof about 254 seconds and 3385 seconds ( 56 minutes) respectively. These treatment times are 3.9 and 2.7 times lower than the usual ones respectively.

Conclusions:Resultsobtained with the proposed model show that the three studied light doses, although all efficient, lead to variable local damages. Defining a reference damage enables the non-optimalparametersfor the current light doses to be refined and the treatment to be more suitable. Furthermore, the model relies on the questionable assumption of unlimited availability of oxygen and further investigation of the change in oxygen availability during treatment could therefore still be carried out to improve the performance and the reliability of the model.

\section{Keywords:Photodynamic Therapy, light doses comparison, protoporphyrin IX, mathematical modeling}

\section{Introduction}

Photodynamic therapy (PDT) is anemerging cancer therapy combining lightof appropriate wavelength, a nontoxic photosensitizer (PS), and sufficient molecular oxygento generate reactive oxygen species and destroy tumors[1,2]. Many reports on photodynamic therapy using 5- aminolevulinic acid (5-ALA-PDT)[3-7] have been published since the early work of Kennedy et al.[8]. 5-ALA is a precursor of the heme biosynthesis and exogenous administration of 5-ALA leads toaccumulation of the photosensitizer protoporphyrin IX (PpIX) preferentially in neoplastic tissues[9].As it can be applied topically for dermatological indications, 5-ALA brings several benefits over other photosensitizers such as porphyrin derivatives, which have to be systemically applied[7]. In dermatology, PDT using 5-ALA or its methyl ester (MAL-PDT) has proven to be an efficient topical treatment for numerous (pre) malignant conditions[5,10]including actinic keratosis (AK)[7,11,12], Bowen's disease $[13,14]$ and superficial basal cell carcinoma[15,16].

Several studies have reported that MAL-PDT with red light using a total light dose of 37 $\mathrm{J} / \mathrm{cm}^{2}$ and a fluence rate of $75 \mathrm{~mW} / \mathrm{cm}^{2}$ is an effective treatment option for AK and results insimilarresponse rates and improved cosmetic outcomes compared with standard therapies[12,17]. However, with these light dose parameters, the treatment appears to be very painful $[18,19]$ and concurrent use of cold air analgesia may be required to relieve discomfort 
and pain [17,18].Recently, Apalla et al.[20] demonstrated that red light PDT using a fluence rate between 25 and $50 \mathrm{~mW} / \mathrm{cm}^{2}$ was as effective inthe treatment of $\mathrm{AK}$ as using a fluence rate of $75 \mathrm{~mW} / \mathrm{cm}^{2}$, but much better tolerated by patients. When using blue light at a dose of 10 $\mathrm{J} / \mathrm{cm}^{2}$ delivered at a fluence rate of $10 \mathrm{~mW} / \mathrm{cm}^{2}$, topical ALA PDT has also demonstrated to be a highly effective and safe treatment for multiple actinic keratoses of the face and scalp [11,21].Finally, PDT of AK using daylight activation has proven to be as effective as, and more manageable in clinical practice than conventional red light PDT [22]. With pain scoressignificantly reduced compared to conventional red light illumination, daylight exposure was also found to be better tolerated and more convenient for the patient.

Looking at these various light doses with similar efficiency but variable tolerability, a mathematical modeling of the PDT process was clearly felt to be necessaryto obtain a better understanding of the process and of the relationship between process parameters and process performance (in terms of efficiency and tolerability) [23-25]. This better understanding should result in an improved determination of the optimal treatment parameters [26,27].

In this paper, we propose to model the PDT process for AK treatmentbased on the study of Farrell et al. [28] and using a skin sample model resulting from the inclusion of an AK to the simplified skin model of Liu et al. [24]. The proposed model involves an iterative procedure alternating between updating the local fluence rate and updating the PpIX absorption coefficient. The local fluence rate is calculated by solvingthe 1D diffusion equation [28,29] while the PpIX absorption coefficient is estimated considering biological elimination and continuous accumulation of the PpIX in the AK as well as photobleaching. Standard models are used for biological elimination and continuous accumulation whereas an original simplified model based on an unlimited availability of oxygen and dependingboth on the local fluence rate and the incident wavelengths is proposed for photobleaching. Finally a photodynamic dose defined as a function of the singlet oxygen molecules generated during the treatment is used to quantify the local damage induced by PDT.

The proposed model was applied with the three most common light doses for PDT of AK:

- Dose 1: red light dose, $632 \mathrm{~nm}, 37 \mathrm{~J} / \mathrm{cm}^{2}, 75 \mathrm{~mW} / \mathrm{cm}^{2}, 500 \mathrm{~s}[30,31]$;

- Dose 2:blue light dose, $417 \mathrm{~nm}, 10 \mathrm{~J} / \mathrm{cm}^{2}, 10 \mathrm{~mW} / \mathrm{cm}^{2}, 1000 \mathrm{~s}[32,33]$; 
- Dose 3:daylight dose, the fluence rate for the daylight was set to the solar spectral irradiance downloaded from [34](this fluence rate was consistent with the one used in Campbell et al. [35]), 9000 s[22];

Analysisof the resulting photodynamic doses allowed for a comparison of the doses in terms of local damages and the determination of a reference local damage, defined as the minimum of the three local damages obtained at the deepest part of the actinic keratosissample. Then, it allowed for the estimation of the treatment times required with the fluence rates of the three above-mentioned light doses to achieve this reference damage to the deepest part of the actinic keratosis sample.

\section{Material}

\section{a. Skin sample model}

As AKs are confined to the epidermis (the basement membrane is intact), the simplified skin sample model we used consists of an epidermis section with a thickness of $100 \mu \mathrm{m}$ ([24])including an AK, designed as an ellipsoid. The epidermis and AK tissues are both assumed to be homogeneous. To account for the thickening of the epidermis generally observed in $\mathrm{AK}$, the diameter in depth of the ellipsoid is set to $150 \mu \mathrm{m}$. According to the curettage usually performed prior to PDT, the skin sample model displayed on Figure 1 is finally assumed.

A primary planar beam with fluence rate $S_{0}$ is assumed to perpendicularly irradiate the surface of the skin sample model (Figure 1).

Let $\vec{z}$ be the beam direction, which is also the depth direction of the skin sample model.

Let $\Omega$ be a cuboid with base surface $d S$ and depth $d z$, located at depth $z$ in the AK (Figure $1)$.

\section{b. Light doses}

Three effective light doses reported in literature [22,30-33] and with parameters summarized in Table 1 are studied. 


\begin{tabular}{|c|c|c|c|}
\hline References & $\begin{array}{l}\text { Moseley et al. [30] } \\
\text { Tyrrell et al. [31] }\end{array}$ & $\begin{array}{c}\text { DUSA } \\
\text { Pharmaceuticals[32] } \\
\text { Warren et al. [33] }\end{array}$ & Wiegell et al.[22] \\
\hline $\begin{array}{c}\text { Fluence rate } \\
\text { spectrum }\end{array}$ & $\begin{array}{c}\text { Gaussian distribution } \\
\text { mean: } 632 \mathrm{~nm} \\
\text { FWHM: } 19 \mathrm{~nm}\end{array}$ & $\begin{array}{c}\text { Gaussian distribution } \\
\text { mean: } 417 \mathrm{~nm} \\
\text { FWHM: } 30 \mathrm{~nm}\end{array}$ & $\begin{array}{c}\text { Solar emission } \\
\text { spectrum } \\
\text { Downloaded from } \\
\text { [34] } \\
\text { Consistent with } \\
\text { Campbell et al. [35] }\end{array}$ \\
\hline Fluence & $37 \mathrm{~J} / \mathrm{cm}^{2}$ & $10 \mathrm{~J} / \mathrm{cm}^{2}$ & I. \\
\hline Exposure time & $500 \mathrm{~s}$ & $1000 \mathrm{~s}$ & $9000 \mathrm{~s}$ \\
\hline Fluence rate & $75 \mathrm{~mW} / \mathrm{cm}^{2}$ & $10 \mathrm{~mW} / \mathrm{cm}^{2}$ & II. \\
\hline
\end{tabular}

Table 1: Description of the three light doses

Usual 3 hours and 30 minutes incubations with MAL under occlusive dressing are assumed for the red and blue light doses and for the daylight dose, respectively.

\section{Method}

\section{a. Local total fluence rate determination}

The local total fluence rate, $\varphi$, at location $\vec{r}$ in the skin sample model, is given by the sum of the local diffuse fluence rate, $\varphi_{d}$, and the local incident fluence rate, $\varphi_{i}$ (equation 1).

$$
\varphi(\vec{r})=\varphi_{d}(\vec{r})+\varphi_{i}(\vec{r})
$$

Due to both the biological elimination of PpIX, the conversion of 5-ALA into PpIX and the photobleaching, the PpIX absorption coefficient and therefore the local total fluence rate change during treatment. Similarly to Farrell et al. [28] based on a PpIX concentration varying only with depth, $z$, below the irradiated surface, we have deduced equation 2 from equation 1: 


$$
\varphi(z)=\varphi_{d}(z)+\varphi_{i}(z)
$$

From Farrell et al. [28] and Carp et al. [29], the local diffuse fluence rate, $\varphi_{d}$, can be expressed using equation 3 :

$$
\varphi_{d}(z)=S_{0}\left\{\frac{b}{\sqrt{\mu_{\text {eff }}(z)}} \exp \left[-\int_{0}^{z} \mu_{\text {eff }}(w) d w\right\rfloor+P(z) \exp \left[-\int_{0}^{z} \mu_{t}^{\prime}(w) d w\right\rceil\right\}
$$

Where:

- The total absorption coefficient, $\mu_{a}$, is the sum of the actinic keratosis absorption coefficient, $\mu_{a, A K}$, and the PpIX absorption coefficient, $\mu_{a, P_{P} I X}$,

- The total transport coefficient, $\mu_{t}^{\prime}$, is the sum of the total absorption coefficient, ${ }_{a}$, and the actinic keratosis reduced scattering coefficient, $\mu_{s, A K}^{\prime}$,

- The effective attenuation coefficient, $\mu_{\text {eff }}$, is defined as $\sqrt{3 \mu_{a}(z) \mu_{t}^{\prime}(z)}$,

- The two parameters, $b$ and $P(z)$, depending on both the optical properties of the actinic keratosis and the boundary conditions at the actinic keratosis surface, are computed as described in Farrell et al. [28].

For a planar beam irradiation, the local incident fluence rate, $\varphi_{i}$, is written in the form of equation $4[28,29]$ :

$$
\varphi_{i}(z)=S_{0} \exp \left[-\int_{0}^{z} \mu_{t}^{\prime}(w) d w\right\rfloor
$$

\section{b. Evolution of thePpIX absorption coefficient}

As three different above mentioned processes affect the PpIX absorption coefficient, the change in the number of PpIX molecules can therefore be expressed as follows (equation 5):

$$
\frac{d M_{P_{P} I X}(t, z)}{d t}=-M_{P_{P D X}, b}(t, z)+M_{P_{p} I X, c}(t, z)-M_{P_{P} I X, p}(t, z)
$$

Where:

- $\quad M_{P P I X}(t, z)$ is the number of PpIX molecules contained in $\Omega$ at time $t$, 
- $\quad M_{P_{p} I X, b}(t, z), M_{P_{P I X}, c}(t, z)$ and $M_{P_{P} I X, p}(t, z)$ are the number of PpIX molecules biologically eliminated, generated by conversion from 5-ALA, and eliminated by photobleaching, respectively, at time $t$.

\section{i. Biological elimination of PpIX}

The biological elimination of PpIX leads to an exponential decay of the number of PpIX molecules such as $M_{P_{p l X}, b}(t, z)$ can be expressed through equation 6:

$$
M_{P p l X, b}(t, z)=\frac{M_{P_{p} I X}(t, z)}{\tau_{b}}
$$

Where $\tau_{b}$ is the time constant for the biological elimination of PpIX for actinic keratosis.

\section{ii. Conversion of 5-ALA into PpIX}

To model the conversion of 5-ALA into PpIX, we use the fluorescence data reported in Wiegell et al. [22]. These data, measured from actinic keratosis within 3hours incubation after MAL application, suggest an exponential increase with time of the number of PpIX moleculesleading to equation 7 :

$$
M_{P P I X, c}(t, z)=\frac{M_{P_{p} I X}(t, z)}{\tau_{c}}
$$

Where $\tau_{c}$ is the time constant for the conversion of 5-ALA into PpIX.

\section{iii. Photobleaching}

As shown by Dysart et al. [36], the change in the concentration of PpIX molecules due to the singlet oxygen-mediated photobleaching can be expressed by a differential equation. This differential equation can be written in term of the number of PpIX molecules, $M_{P_{P} I X}(t, z)$, (equation 8):

$$
M_{P_{P l X}, p}(t, z)=\frac{\kappa}{\aleph \times d S \times d z} \times M_{P_{p} I X}(t, z) \times M_{{ }_{1}}(t, z)
$$

Where:

- $\kappa$ is the bimolecular rate constantfor the reaction of singlet oxygen with PpIX,

- $\leqslant$ is the Avogadro number,

- $M_{{ }^{\circ}}(t, z)$ is the number of singlet oxygen molecules contained in $\Omega$ at time $t$. 
Thechange in the number of singlet oxygen molecules contained in $\Omega$ at time $t$ can be expressed as (equation 9):

$$
\frac{d M_{{ }_{{ }_{o}}}(t, z)}{d t}=+M_{{ }_{o}}^{+}(t, z)-M_{{ }_{o}}^{-}(t, z)
$$

Where:

- $\quad M_{{ }_{o}}^{+}(t, z)$ is the number of PpIX molecules generated in $\Omega$ at time $t$ when the PpIX molecules, excited by absorption of photons, return to the ground state,

- $\quad M_{{ }_{o}}^{-}(t, z)$ is the number of PpIX molecules consumed in $\Omega$ at time $t$.

Using the first-order approximation of the derivative, equation 10 is obtained:

$$
M_{\mathrm{I}_{o}}(t, z)=M_{\mathrm{I}_{o}}(t-d t, z)+d t \times M_{\mathrm{I}_{o}}^{+}(t, z)-d t \times M_{\mathrm{I}_{o}}^{-}(t, z)
$$

According to the short lifetimes of the excited states of the PpIX ( nanoseconds), simultaneity between the absorption of a photon and the subsequent production of singlet oxygen molecules is assumed such that $M_{i_{o}}^{+}(t, z)$ can be estimatedas follows (equation 11):

$$
M_{{ }_{0} o}^{+}(t, z)=\int_{\tilde{\lambda}}\left\{\gamma_{\tilde{\lambda}} \times \frac{\varphi(t, z, \tilde{\lambda}) \times d S}{E_{\tilde{\lambda}}} \times \mu_{a, P_{p} I X}(t, z, \tilde{\lambda}) \times d z\right\} d \tilde{\lambda}
$$

Where:

- The (dimensionless) singlet oxygen quantum yield, $\gamma_{\tilde{i}}$, is the number of molecules of singlet oxygen molecules generated for each photon of wavelength $\tilde{\lambda}$ absorbed by a PpIX molecule when the PDT process is not limited by the availability of oxygen concentration,

- Computed from the local total fluence rate reaching $d S$ at time $t, \varphi(t, z, \tilde{\lambda})$, and from the energy of a photon of wavelength $\tilde{\lambda}, E_{\tilde{\lambda}}$, the term $\frac{\varphi(t, z, \tilde{\lambda}) \times d S}{E_{\tilde{\lambda}}}$ represents the number of photons of wavelength $\tilde{\lambda}$ reaching $d S$ per unit of time.

Moreover, regarding the short singlet oxygen lifetime in biological media ( hundredths of microseconds [36]) compared to the interval of time $d t$ usually used for computations ( hundred microseconds), all the singlet oxygen molecules, present in $\Omega$ at time $t-d t$, 
$M_{{ }_{1}}(t-d t, z)$, are assumed to be consumed during $d t$ such that $M_{\mathrm{o}_{o}}^{-}(t, z)$ can be approximated by $\frac{M_{{ }^{\prime} O}(t-d t, z)}{d t}$ leading to equation 12 :

$$
M_{{ }^{\prime} o}(t, z)=d t \times M_{{ }_{o}}^{+}(t, z)=d t \times \int_{\tilde{\lambda}}\left\{\gamma_{\tilde{\lambda}} \times \frac{\varphi(t, z, \tilde{\lambda}) \times d S}{E_{\tilde{\lambda}}} \times \mu_{a, p_{p} I X}(t, z, \tilde{\lambda}) \times d z\right\} d \tilde{\lambda}
$$

Finally, the number of PpIX molecules eliminated by photobleaching can be obtained through equation 13:

$$
\begin{aligned}
& M_{P_{P} I X, p}(t, z) \\
& \quad=\frac{\kappa}{\aleph \times d S \times d z} \times M_{P_{p} I X}(t, z) \times d t \times \int_{\tilde{\lambda}}\left\{\gamma_{\tilde{\lambda}} \times \frac{\varphi(t, z, \tilde{\lambda}) \times d S}{E_{\tilde{\lambda}}} \times \mu_{a, P_{P} I X}(t, z, \tilde{\lambda}) \times d z\right\} d \tilde{\lambda} \\
& \quad=\frac{\kappa}{\aleph} \times M_{P_{P} I X}(t, z) \times d t \times \int_{\tilde{\lambda}}\left\{\gamma_{\tilde{\lambda}} \times \frac{\varphi(t, z, \tilde{\lambda})}{E_{\tilde{\lambda}}} \times \mu_{a, P_{P} I X}(t, z, \tilde{\lambda})\right\} d \tilde{\lambda}
\end{aligned}
$$

\section{iv. Overall evolution}

Inserting equations 6, 7 and 13 into equation 5 gives equation 14:

$$
\frac{d M_{P_{P I X}}(t, z)}{d t}=M_{P_{p l X}}(t, z) \times\left\{-\frac{1}{\tau_{b}}+\frac{1}{\tau_{c}}-\frac{\kappa}{\aleph} \times d t \times \int_{\tilde{\lambda}}\left\{\gamma_{\tilde{\lambda}} \times \frac{\varphi(t, z, \tilde{\lambda})}{E_{\tilde{\lambda}}} \times \mu_{a, P_{p l X}}(t, z, \tilde{\lambda})\right\} d ;\right.
$$

Based on the relation $\mu_{a, P_{P} I X}(t, z, \lambda)=\varepsilon_{P_{P I X}}(\lambda) \times C_{P_{P} I X}(t, z)=\varepsilon_{P_{P I X}}(\lambda) \times \frac{M_{P_{P} I X}(t, z)}{\aleph \times d S \times d z}$ where $\varepsilon_{P_{P I X}}(\lambda)$ and $C_{P_{P} I X}(t, z)$ are the PpIX molar extinction coefficient for wavelength $\lambda$ and the concentration at depth $z$ and time $t$, respectively, equation 14 leads to equation 15:

$$
\begin{aligned}
& \frac{d \mu_{a, P_{p} I X}(t, z, \lambda)}{d t}= \\
& \quad \mu_{a, P_{p} I X}(t, z, \lambda) \times\left\{-\frac{1}{\tau_{b}}+\frac{1}{\tau_{c}}-\frac{\kappa}{\aleph} \times d t \times \int_{\tilde{\lambda}}\left\{\gamma_{\tilde{\lambda}} \times \frac{\varphi(t, z, \tilde{\lambda})}{E_{\tilde{\lambda}}} \times \mu_{a, P_{p} I X}(t, z, \tilde{\lambda})\right\} d \tilde{\lambda}\right.
\end{aligned}
$$

\section{c. The photodynamic dose}

Because damage induced by PDT is a result of the generation of singlet oxygen, the photodynamic dose can be defined as the total cumulative singlet oxygen produced during 
treatment time, denoted $T$. From equation 11, it therefore follows (equation 16):

$$
P D(z)=\int_{0}^{T} M_{{ }^{\prime}}^{+}(t, z) d t=\int_{0}^{T} \int_{\tilde{\lambda}}\left\{\gamma_{\tilde{\lambda}} \times \frac{\varphi(t, z, \tilde{\lambda}) \times d S}{E_{\tilde{\lambda}}} \times \mu_{a, P_{p} I X}(t, z, \tilde{\lambda}) \times d z\right\} d \tilde{\lambda} d t
$$

Using the sampling times $\left\{t_{i}=i \times d t\right\}_{0 \leq t_{i} \leq T}, P D(z)$ can be approximated as in equation 17:

$$
P D(z) \approx \sum_{t_{i}}\left\{d t \times \int_{\tilde{\lambda}}\left\{\gamma_{\tilde{\lambda}} \times \frac{\varphi\left(t_{i}, z, \tilde{\lambda}\right) \times d S}{E_{\tilde{\lambda}}} \times \mu_{a, P_{P} I X}\left(t_{i}, z, \tilde{\lambda}\right) \times d z\right\} d \tilde{\lambda}\right\}
$$

The calculation of $P D(z)$ therefore requires the determination of boththe PpIX absorption coefficient and the local total fluence rate as treatment progresses.

Assuming an initial PpIX absorption coefficient, $\mu_{a, P_{P} I X}(0, z, \lambda)$, the initial local total fluence rate, $\varphi(0, z, \lambda)$, at any point of the skin model (Figure 1) can be calculated from equations 2 to 4. The PpIX absorption coefficient at time $t_{1}=d t$ can then be obtained considering the following approximation of equation 15 (equation 18):

$$
\begin{aligned}
& \mu_{a, P_{P} I X}(t+d t, z, \lambda)=\mu_{a, P_{P I X}}(t, z, \lambda) \\
& \quad+d t \times \mu_{a, P_{P} I X}(t, z, \lambda) \times\left\{-\frac{1}{\tau_{b}}+\frac{1}{\tau_{c}}-\frac{\kappa}{\aleph} \times d t \times \int_{\tilde{\lambda}}\left\{\gamma_{\tilde{\lambda}} \times \frac{\varphi(t, z, \tilde{\lambda})}{E_{\tilde{\lambda}}} \times \mu_{a, P_{P} I X}(t, z, \tilde{\lambda})\right\} d \tilde{\lambda}\right.
\end{aligned}
$$

From this new PpIX absorption coefficient, the new local total fluence rate, $\varphi\left(t_{1}, z, \lambda\right)$, is calculated. The process is reiterated to calculate all the necessary PpIX absorption coefficients and local total fluence rates.

\section{d. Initialization}

According to the initial PpIX absorption coefficient, similarly to Liu et al. [24], an initial exponential distribution of PpIX with depth, related to the progressive skin penetration of 5ALA, is assumed (equation 19):

$$
\mu_{a, P P I X}(0, z, \lambda)=\mu_{a, P_{P} I X}(0,0, \lambda) \times \exp (-\eta z)
$$

Where $\eta$ is the depth decay constant. 
Moreover, from the above-mentioned relation $\mu_{a, P_{P I X}}(t, z, \lambda)=\varepsilon_{P_{P I X}}(\lambda) \times C_{P_{P} I X}(t, z)$, equation 19 becomes equation 20 :

$$
\mu_{a, P P I X}(0, z, \lambda)=\varepsilon_{P P D X}(\lambda) \times C_{P P I X}(0,0) \times \exp (-\eta z)
$$

\section{e. Parameters specification}

The optical properties for epidermis are derived from the data obtained by Salomatina et al [37] from normal human skin and the ones for actinic keratosis from the data reported in Garcia-Uribe et al. [38].

Regarding the time constant for the biological elimination of PpIX, denotedabove as $b$ (equation 6), we used the value of $1.29 \mathrm{~h}$ obtained by Star et al. [39] for normal human epidermis.

From the actinic keratosis data reported in Wiegell et al. [22], the time constant for the conversion of 5-ALA into PpIX, $\tau_{c}$ (equation 7), is deduced to be $1.1575 \mathrm{~h}$, which is consistent with previously published values $[39,40]$.

Regarding the bimolecular rate constant, $\kappa$ (equation 8 ), we use the value of $5.3 \times 10^{9} \mathrm{l} / \mathrm{mol} / \mathrm{s}$ reported in [41] as the bimolecular rate constant for quenching of protoporphyrin IX by Formula Ilai.

According to Wilkinson et al. [42] and Fernandez et al. [43], the singlet oxygen quantum yield for PpIX, $\gamma_{\lambda}$ (equation 11), is set to 0.56 for all $\lambda$.

For the sake of consistency in equation 11 (the number of PpIX molecules in their singlet excited state can not exceed the number of available PpIX molecules), the time increment, $d t$, is set to $1 \times 10^{-5} \mathrm{~s}$.

Based on the ratio of PpIX concentration at $0.2 \mathrm{~mm}$ to that on the surface of about $81 \%$ (respectively, 63\%) obtained by Star et al. [39] for normal human epidermis after 3hours (respectively, 30 minutes) ALA administration, we deduce that the depth decay constant $\eta$ (equation 19) is equal to $1.05 / \mathrm{mm}$ (respectively, $2.31 / \mathrm{mm}$ ) for the red and blue light doses 
(respectively, for the daylight dose) with theabove assumed 3 hours (respectively, 30 minutes) incubation.

Regarding the initial concentration at the skin surface in equation $20, C_{P_{P} I X}(0,0)$, we use the value $11.8 \mathrm{pmol} / \mathrm{ml}$ obtained by Smits et al. [44]from 11 patients with AK incubated with $20 \%$ ALA for 3hours. This value suitable for the red and blue light doses with 3hours incubation is not appropriate for the daylight dose with 30 minutes incubation. Based on a fluorescence intensity after 30 minutes incubation graphically deduced to be approximately 10 times lower than the one after 3hours incubation from Wiegell et al. [22] and Christiansen et al. [45], we set the initial concentration at the skin surface for the daylight dose to 1.18 $\mathrm{pmol} / \mathrm{ml}$.

The PpIX molar extinction coefficients, $\left\{\varepsilon_{P_{p I X}}(\lambda)\right\}_{2}$, are estimated from the PpIX absorption spectrum, $\left\{\mu_{a, P P I X, C R A N}(\lambda)\right\}_{\lambda}$, measured by the Research Center for Automatic Control of Nancy (CRAN)from a PpIX concentration $C_{P P I X, C R A N}$.The estimates,derivedfrom the relation $\mu_{a, P P I X, C R A N}(\lambda)=\varepsilon_{P_{P I X} I X}(\lambda) \times C_{P_{P} I X, C R A N}$, are deduced usingthe value of $1.24 \times 10^{5} 1 / \mathrm{mol} / \mathrm{cm}$ for $\varepsilon(405 \mathrm{~nm})$ reported in Natarajan et al. [46](equation 21):

$$
\varepsilon_{P P I X}(\lambda)=\frac{\mu_{a, P P I X, C R A N}(\lambda)}{C_{P_{P I X}, C R A N}}=\frac{\mu_{a, P P I X, C R A N}(\lambda)}{\mu_{a, P P I X, C R A N}(405 \mathrm{~nm})} \times \varepsilon_{P P I X}(405 \mathrm{~nm})
$$

The published values for the model parameters that do not depend (respectively, that depend) on the light source are listed in Table 2(respectively, in Table 3).

\begin{tabular}{clc}
\hline Parameters & Value & Reference(photosensitizer,cells) \\
\hline $\begin{array}{c}\text { Optical properties } \\
\text { for epidermis }\end{array}$ & III. & Salomatina et al [37] \\
\hline $\begin{array}{c}\text { Optical properties } \\
\text { for actinic keratosis }\end{array}$ & IV. & Garcia-Uribe et al. [38] \\
\hline$\tau_{b}$ & $1.3 \mathrm{~h}$ & Star et al. [39](PpIX, normal human \\
epidermis)
\end{tabular}




\begin{tabular}{ccc}
\hline$\kappa$ & $\begin{array}{c}2.3 \times 10^{7} \\
1 / \mathrm{mol} / \mathrm{s}\end{array}$ & keratosis) \\
\hline$\gamma_{\lambda}$ & 0.56 & Wilkinson et al. [42] (PpIX) \\
& & Fernandez et al. [43](PpIX) \\
\hline$d t$ & $1 \times 10^{-5} \mathrm{~s}$ & V. \\
\hline$\varepsilon(405 \mathrm{~nm})$ & $1.24 \times 10^{5}$ & Natarajan et al. [46] (PpIX) \\
\hline
\end{tabular}

Table 2: Specification of the model parameters not depending on the light sources. For each reference, the photosensitizer and cells for which the values were obtained are reported into brackets

\begin{tabular}{cccc}
\hline Parameters & Light source & Value & Reference (photosensitizer, cells) \\
\hline & Red and & & Star et al. [39](PpIX, normal human \\
blue light & $1.05 / \mathrm{mm}$ & epidermis) \\
doses & & \\
\hline
\end{tabular}

Daylight dose $\quad 2.31 / \mathrm{mm}$

\begin{tabular}{cccc}
\hline & $\begin{array}{c}\text { Red and } \\
\text { blue light } \\
\text { doses }\end{array}$ & $\begin{array}{c}11.8 \\
\text { pmol/ml }\end{array}$ & $\begin{array}{c}\text { Smits et al. [44](PpIX, actinic } \\
\text { keratosis) }\end{array}$ \\
\cline { 2 - 4 } & Daylight dose & $1.18 \mathrm{pmol} / \mathrm{ml}$ & $\begin{array}{c}\text { Smits et al. [44](PpIX, actinic keratosis) } \\
\text { Wiegell et al. [22](PpIX, actinic keratosis) }\end{array}$
\end{tabular}

Table 3: Specification of the model parameters depending on the light sources. For each reference, the photosensitizer and cells for which the values were obtained are reported into brackets

\section{Applications}

By down-sampling the skin model into $\Omega$ cuboids with $d S=10 \mu \mathrm{m} \times 10 \mu \mathrm{m}$ and $d z=10 \mu \mathrm{m}$ as partially illustrated in Figure 1, the photodynamic doses for each cuboid are computed for the three light dosesaccording to equation 17. 
We assume that, whatever the position in the AK of the skin sample model, the three obtained photodynamic doses are lethal for any cancer cells.It follows that the minimum of the three photodynamic doses at the deepest point of the AK, the darkest cuboid of thestack of cuboids shownin Figure 1, that we denote by $P D_{r e f}$, is also assumed to be lethal.

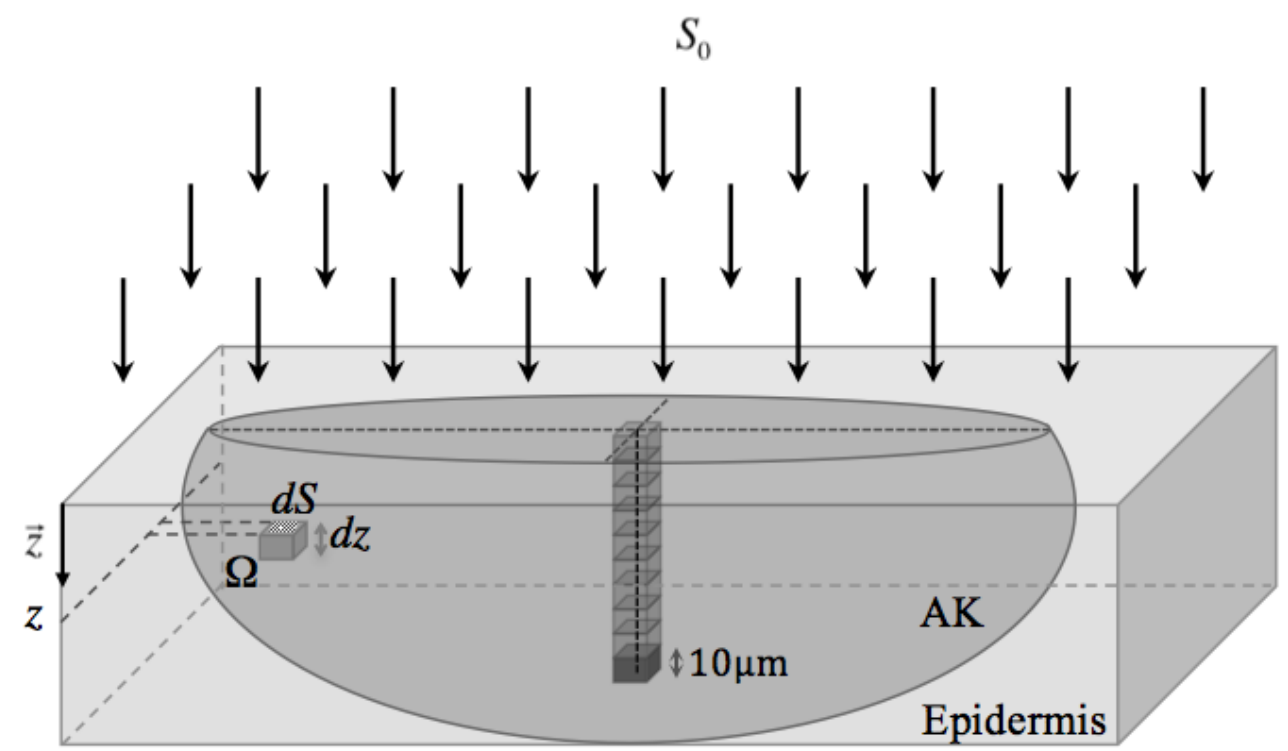

Figure 1:Representation of the skin sample model perpendicularly irradiated by a planar beam $S_{0}$. The sampling of the skin sample model is partially illustratedand the deepest point of the AKis identified as the darkest cuboid of the central stack of cuboids.

Based on the assumption that, whatever the light dose, a photodynamic dose equal to $P D_{r e f}$ is sufficient to destroy any cancer cells, the treatment times and therefore the light doses required with the three light sources and their corresponding fluence rates reported in Table 1to obtain a photodynamic dose equal to $P D_{\text {ref }}$ are computed.

All the computations were performed using a Matlab ${ }^{\mathrm{TM}}$ program on a standard personal computer (Intel Xeon CPU E3-1240 V2 3.40 GHz-8Go of RAM-Windows 764 bits).

\section{Results}

The photodynamic doses obtained inthe skin sample model for the three different light doses are presented according to the central cross section of the skin sample modelin Figure 2 and along the above-defined central stack of cuboids (Figure 1) in Figure 3. 


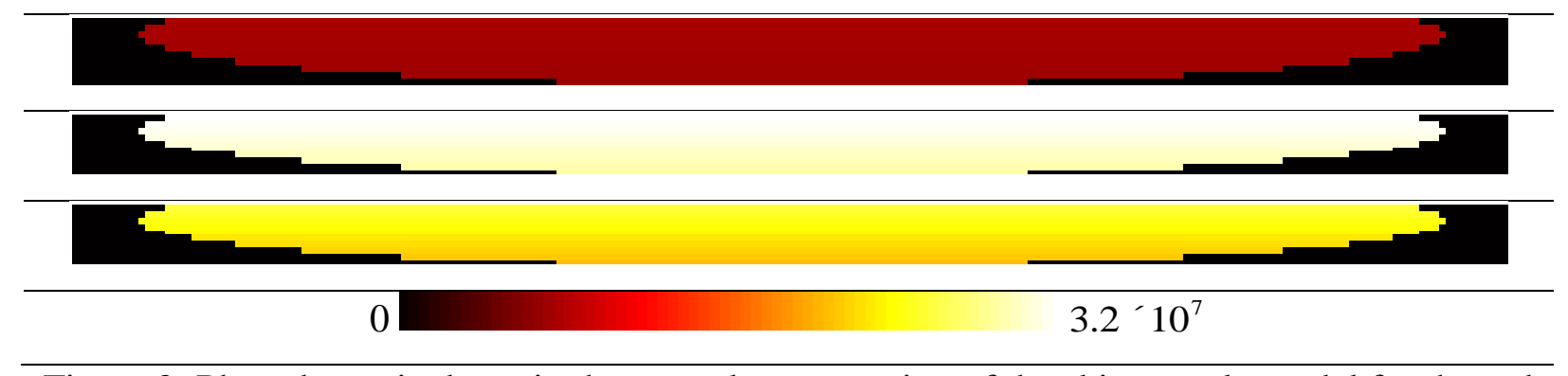

Figure 2: Photodynamic doses in the central cross section of the skin sample model for the red light dose (row 1), the blue light dose (row 2) and the daylight dose (row 3). The doses are displayed, using the hot look-up table (row 4), in percent of the overall maximum photodynamic dose of $3.210^{7}$ that wasobtained at the skin surface with the blue light dose (see the y-intercept of the blue curve in Figure 3.a).

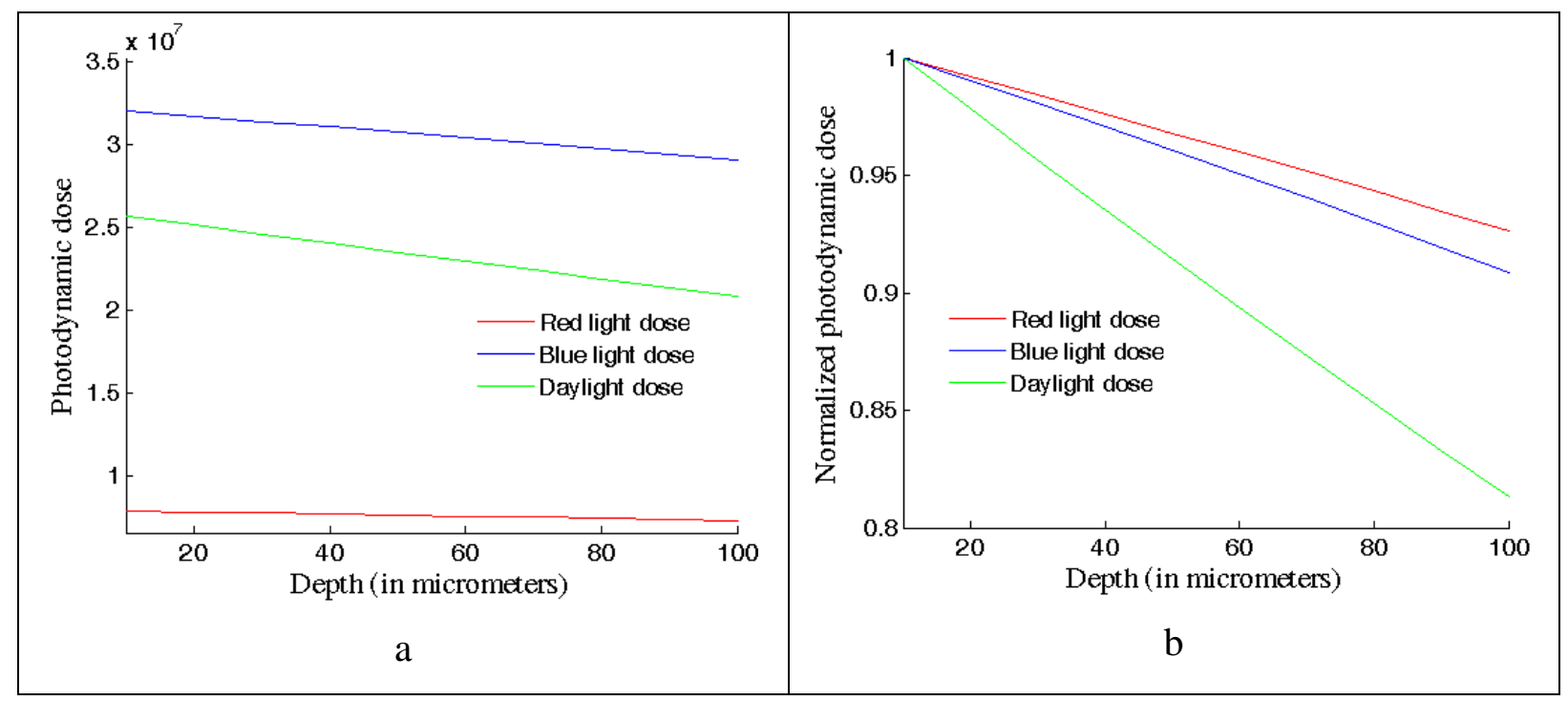

Figure 3: Depth evolution of both the photodynamic doses (a) and normalized photodynamic doses (b) for the red light dose (red curve), the blue light dose (blue curve) and the daylight dose (green curve) along the central stack of cuboids of the skin sample model defined in Figure 1. For each light source, the normalized photodynamic doses are obtained by dividing the photodynamic doses by the photodynamic dose at $10 \mu$ mdepth.

From Figure 3, the depth evolution of boththe photodynamic doses and normalized photodynamic doses for the red light dose (red curve), the blue light dose (blue curve) and the daylight dose (green curve) seems to be linear. With a slope of $-2.1 \times 10^{-3}$ from the linear regression, the depth evolution of the normalized photodynamic dose for the daylight dose is approximately twice (respectively, 2.5 times) as rapid as the one for the blue light dose 
(respectively, the red light dose) with a slope of $-5.82 \times 10^{-4}$ (respectively, $-6.46 \times 10^{-4}$ ) (Figure 3.b). From the last two slope values, the normalized photodynamic dose for the red light dose is found to decrease in depth approximately 1.1 times as fast as the blue light dose one.

Figure 4 showsthe time evolution of the cumulative singlet oxygen producedat the deepest point of the AKfor the three different light doses. This cumulative parameter was approximated at the above defined sampling times similarly to equation 17 .

From the linear regression of the curves of Figure 4, the increase rate (defined as the slope of the linear regression) of the cumulative singlet oxygen produced for the blue light dose with a fluence rate 7.5 times lower than the one for the red light dose is found to be almost twice as high as the one for the red lightdose. The linear regression also shows that the increase rate of the cumulative singlet oxygen produced for the daylight dose is a little less than seven times lower than the one for the red light dose.

From the three photodynamic doses of $5.67 \times 10^{6}, 1.45 \times 10^{7}$ and $2.1 \times 10^{7}$ obtained at the deepest point of the AK with the red light dose, the blue light dose and the daylight dose, respectively (given by the last point of the curves in Figures3.a and 4), the minimum one denoted $P D{ }_{\text {ref }}$ was determined to be thered one.

From Figure 4, using the blue light with a fluence rate of $10 \mathrm{~mW} / \mathrm{cm}^{2}$ (Table 1), a treatment time of about 254 seconds, which correspond to a light dose of $2.54 \mathrm{~J} / \mathrm{cm}^{2}$, is required to obtain a photodynamic dose equal to $P D_{\text {ref }}$ at the deepest part in the AK.For the daylight dose(Table 1), a photodynamic dose equal to $P D_{r e f}$ is achieved at the deepest part of the $\mathrm{AK}$ using exposure time of 3385 seconds. 


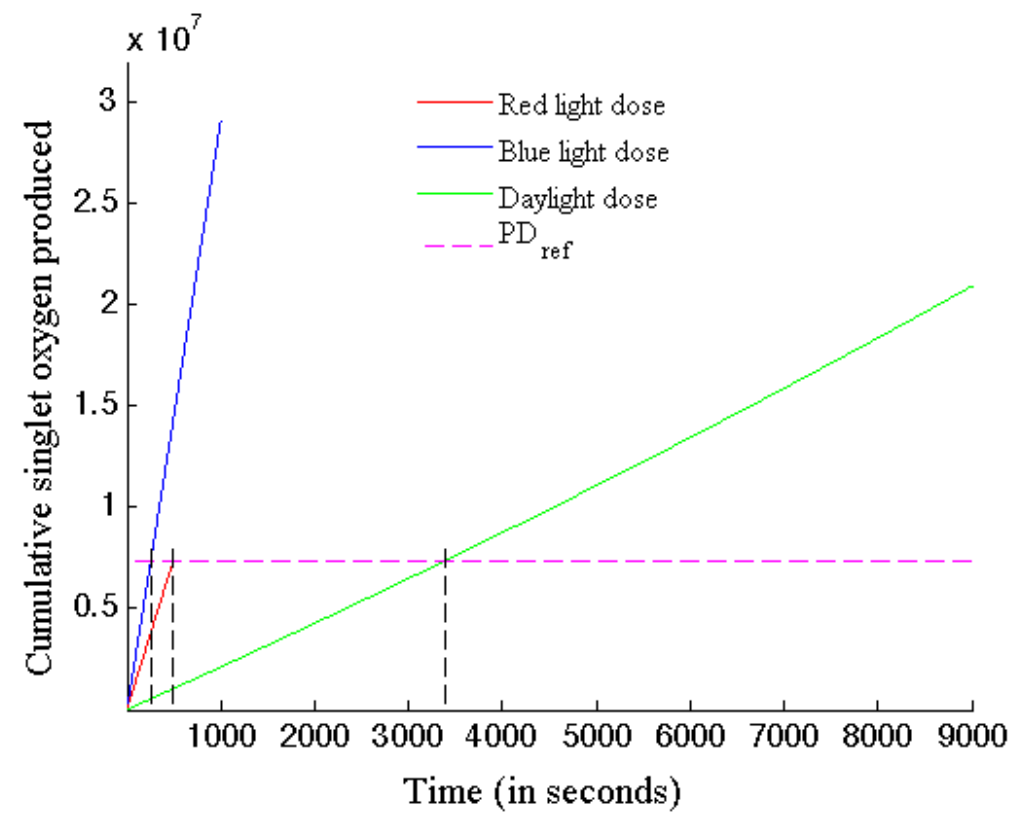

Figure 4: Time evolution of the cumulative singlet oxygen produced for the red light dose (red curve), the blue light dose (blue curve) and the daylight dose (green curve) at the deepest point of the AK. The pink dashed line was used to determine the treatment times required with the blue light and the daylight as described in Table 1 to obtain a photodynamic dose at the deepest part of the AK equal to $P D_{\text {ref }}$.

Figure 5 showsexamples of the evolutionin time of the PpIX absorption coefficient obtained using the proposed model (equation 15).The corresponding evolutions in time of the PpIX absorption coefficient obtained by replacing the proposed model for the photobleaching (equation 13) by the common first order photobleaching modelare also depicted in Figure 5, for information purposes only.The exponential decay of the number of PpIX molecules assumed by the first order photobleaching model (equation 22.a)[23,28,35] leads to equation (22.b) in place of equation (15):

$$
\left\{\begin{array}{c}
M_{P_{P} I X, p}(t, z)=-\int_{\tilde{\lambda}} \beta_{\tilde{\lambda}} \times \varphi(t, z, \tilde{\lambda}) \times M_{P_{P} I X}(t, z) d \tilde{\lambda} \\
\mu_{a, P_{P} I X}(t+d t, z, \lambda)=\mu_{a, P_{P} I X}(t, z, \lambda) \\
\quad+d t \times \mu_{a, P_{P} I X}(t, z, \lambda) \times\left\{-\frac{1}{\tau_{b}}+\frac{1}{\tau_{c}}-\int_{\tilde{\lambda}} \beta_{\tilde{\lambda}} \times \varphi(t, z, \tilde{\lambda}) d \tilde{\lambda}\right\}
\end{array}\right.
$$

Where the photobleaching dose constant parameters $\left\{\beta_{\tilde{\lambda}}\right\}_{\tilde{\lambda}}$ are set to $0.05 \mathrm{~cm}^{2} / \mathrm{J}$ for all $\lambda[28]$. 


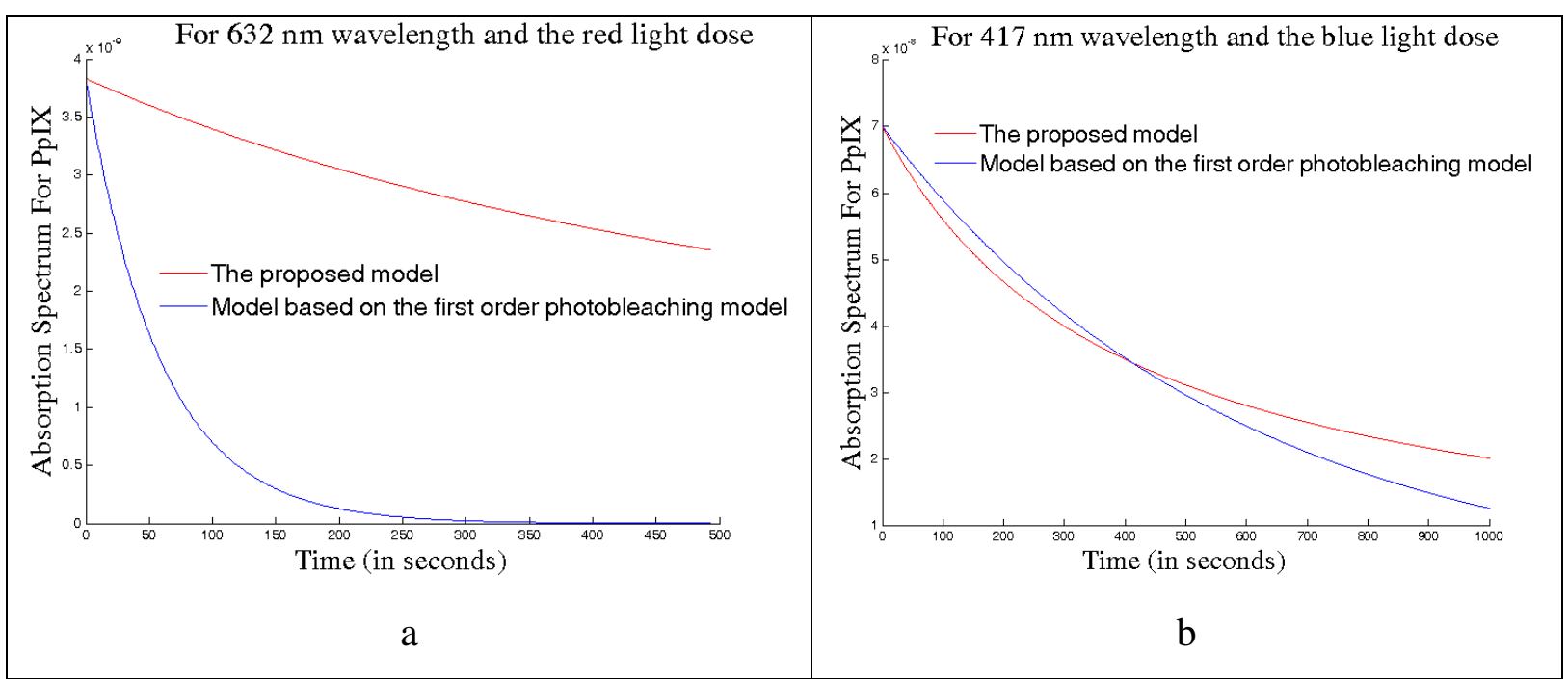

Figure 5: Time evolution of the PpIX absorption coefficient at the deepest point of the AK obtained at wavelength $632 \mathrm{~nm}$ with the red light dose (a) and at wavelength $417 \mathrm{~nm}$ with the blue light dose (b). The red curves represent the results obtained using the proposed model (equation 15) while the blue ones represent the results obtained by considering the first order photobleaching model $[23,28,35]$ (equation 22.b).

From Figure 5.b (for the $417 \mathrm{~nm}$ wavelength and the blue light dose), the red time evolution curve obtained using the proposed model and the blue one obtained by considering the first order photobleaching model follow somewhat similar trends. This similarity is not found for the $632 \mathrm{~nm}$ wavelength and the red light dose (Figure 5.a). This may be explained by the use of a single value for all the photobleaching dose constant parameters $\left\{\beta_{\tilde{\lambda}}\right\}_{\tilde{\lambda}}$ that may be more appropriate for specific wavelengths. Furthermore, we can note a very rapid decrease of the blue curve in Figure 5.a, which presupposes a very (maybe too?) rapid consumption of PpIX.

\section{Discussion}

In this paper, three light doses commonly used in the PDT treatment of actinic keratosis are compared using a mathematical modeling of the PDT process: the red light dose (632 nm, 37 $\mathrm{J} / \mathrm{cm}^{2}, 75 \mathrm{~mW} / \mathrm{cm}^{2}, 500 \mathrm{~s},[30,31]$ the blue light dose $\left(417 \mathrm{~nm}, 10 \mathrm{~J} / \mathrm{cm}^{2}, 10 \mathrm{~mW} / \mathrm{cm}^{2}, 1000 \mathrm{~s}\right.$, [33]) and the daylight dose (9000 s, [22]) (Table 1).

The comparison is performed using a skin sample model consisting of an epidermis section with a thickness of $100 \mu \mathrm{m}$ including an AK designed as a partial ellipsoid with $150 \mu \mathrm{m}$ thick (Figure 1). Although similar high response rates have been reported for PDT treatment of AK using the three above introduced lights doses $[12,21,22,47]$ the deeper tissue penetration of 
red light compared to light with shorter wavelengths is known to make the red light dose more appropriate for the PDT treatment of thick lesions and deeper targets [48]. Nonetheless, regarding the thin actinic keratosis of the skin sample model used in this study, the blue light and the daylight can reasonablybe assumed to allow sufficient tissue penetration to make the blue light and daylight doses efficient.

To perform the comparison of the three light doses, a photodynamic dose defined as the total cumulative singlet oxygen produced during treatmentis introduced(equations 16 and 17). This photodynamic dose depends on the local total fluence rate, obtained by the sum of the local diffuse fluence rate and the local incident fluence rate[28,29] (equations 1 to 4 ), and on the PpIX absorption coefficient. The three common kinds of changes in the PpIX absorption coefficient, namely the biological elimination of PpIX, the conversion of 5-ALA into PpIX and the photobleaching, are considered in the model (equations 5 to 15). While usual exponential models are used for the biological elimination and the 5-ALA conversion, we proposed a new model for the photobleaching. The two commonly used models for photobleaching that arethe first order photobleaching one in which PpIX is bleached exponentially by local total fluence rate[23,28] and the second order one using standard photochemical reaction kinetics[24,25] seem not to be appropriate for the present study. Regarding the first order photobleaching model, few published values are available for the involved photobleaching dose constant parameter and these values that are mainly obtained with a red light illumination[23,35] may not be suitable for the blue light and daylight illuminations and may therefore bias the comparison. The photobleaching model we proposed allows us to get rid of this photobleaching dose constant parameter and to make explicit the photobleaching dependence on wavelength (equation 13).Moreover itis adapted to the multispectral case and involves relatively few parameterscompared to the complex second order photobleaching model.

The proposed model has been developed so as to involve only parameters for which empirical data are available and has therefore required some assumptions and simplifications especially inthe change in the number of singlet oxygen molecules (equations 9 to 12). These approximations are acceptable in the context of a comparison of light doses and may, in the current trends of low or blue dose, be suitable to define an experimental protocol to determine optimal treatment parameters. 
All the parameters (equations 1 to 20) are set topublished values, which were obtained with PpIX and with either normal human epidermis or AK, except forthe bimolecular rate constant (Table 2).

A first limitation of our model is the fact that the PpIX concentration is assumed to vary only with depthbelow the irradiated surface as in [28]. Nonetheless, given the very small thickness of the skin sample model $(100 \mu \mathrm{m})$, this assumption can be tolerated. A second limitation concerns the assumption ofunlimited availability of oxygen (oxygen depletion due to photobleaching is not incorporated in the model). This assumption,made through the singlet oxygen quantum yield in equation 11, can be considered reasonable under light illumination with low fluence rates[49,50], which is not the case for the red light dose.However, it is difficult to ascertain how the singlet oxygen quantum yield, $\gamma_{\lambda}$, would change during treatment and due to the lack of well-established empirical data and the variations of intrinsic parameters, we assumed constant $\gamma_{\lambda}$.According to [51], this assumption furthermore seems to be consistent within the present study in which the skin sample model consists of an epidermis section with a thickness of $100 \mu \mathrm{m}$ including an AK. In fact, Stücker et al [51] have reported that the upper skin layers to a depth of $0.25-0.40 \mathrm{~mm}$ (including therefore the epidermis layer) are almost exclusively supplied by diffused oxygen from the atmosphere, whereas the oxygen transport by blood capillaries extending to the upper layers of the dermis has a minor influence. It follows that the unlimited source of atmospheric oxygen allows unlimited oxygen availability in the skin sample model to be reasonably assumed.

Using red light and standard dose (fluence, $37 \mathrm{~J} / \mathrm{cm}^{2}$; fluence rate, $75 \mathrm{~mW} / \mathrm{cm}^{2}$; exposure time, $500 \mathrm{~s}$; [30,31]) a photodynamic dose of about $5.67 \times 10^{6}$ was obtained at the deepest part of the AK (i.e., at $95 \mu \mathrm{m}$ from the skin surface). For a standard blue light dose (light dose, 10 $\mathrm{J} / \mathrm{cm}^{2}$; fluence rate, $10 \mathrm{~mW} / \mathrm{cm}^{2}$; exposure time, $1000 \mathrm{~s}$; [32,33]), a photodynamic dose of about $1.45 \times 10^{7}$ was estimated at the deepest part of the AK. With the daylight dose (exposure time, $9000 \mathrm{~s}$; [22,34]), a photodynamic dose of about $2.1 \times 10^{7}$ was obtained at the deepest part of the AK.

The minimum of these three photodynamic doses, which were considered as sufficiently lethal for AK cancer cells, was therefore obtained with the red light dose (Figure 4). The maximum of these three photodynamic doses was the blue one. This result can be explained 
by thesmallthickness of tissue to be treated $(100 \mu \mathrm{m})$ that makes the above mentioneddeeper tissue penetration of red light compared to light with shorter wavelengths useless and by the better match between the absorption spectrum of the PpIX and the blue light spectrum (PpIX has its largest absorption peak in the blue region). The daylight photodynamic dose was close to the blue one that can be supported by the fact that all the PpIX absorption peaks are within the daylight spectrum.

The treatment time required with the blue light and a fluence rate of $10 \mathrm{~mW} / \mathrm{cm}^{2}$ (Table 1) to obtain a photodynamic dose equal to the red one at the deepest part of the AK was then estimated to be 254 seconds, which is equivalent to a quarter of the usual value of 1000 seconds $[30,31]$. This exposure time corresponds to a light dose of $2.54 \mathrm{~J} / \mathrm{cm}^{2}$.With daylight (Table 1), about 3385 seconds were required to obtain a photodynamic dose equivalent to the red one.A reduction of about $62 \%$ is found between these 3385 seconds and the 9000 seconds reported in [22]. These results tend to highlight that the usual light doses (Table 1) are probably not well adapted and that thetreatment parameters could be better determined to obtain a similar efficiency but animproved tolerability and a more manageable clinical practice (reduction in bed occupancy).

\section{Conclusion}

In this paper, we have proposed an original mathematical model for the photodynamic treatment of actinic keratosis. Applied with the three most common light doses reported in the literature, this model allows 1) a comparison of the local damage at the deepest part of the AK and 2) a comparison of the treatment times required to carry the same local damage to the deepest part of the AK to be made. These comparisons demonstrated that an optimization of the light doses parameters could lead to a similarly efficient and more suitable treatment. 


\section{ACKNOWLEDGMENT}

The authors would like to thank A. N. Yaroslavsky from the Harvard Medical School, Massachusetts General Hospital, Wellman Center for Photomedicine, Boston, USA for providing the optical properties of epidermis used in this paper. The authors would like to thanks A. Garcia-Uribe from Department of Biomedical Engineering, Washington University in St. Louis, St. Louis, Missouri and from Department of Electrical and Computer Engineering, Texas A\&M University, College Station for granting access to the optical properties of actinic keratosis used in the experiments. The authors would like to thank $\mathrm{C}$. Lavogiez from the Department of Dermatology, University Hospital, Lille, France for her cooperation in this study.

\section{References}

[1] Castano AP, Demidova TN, and Hamblin MR, "Mechanisms in photodynamic therapy: part one-photosensitizers, photochemistry and cellular localization", Photodiagnosis and Photodynamic Therapy, 1, 4, 279-293, 2004.

[2] Plaetzer K, Krammer B, Berlanda J, Berr F, and Kiesslich T, "Photophysics and photochemistry of photodynamic therapy: fundamental aspects.", Lasers in Medical Science, 24, 2, 259-268, 2009.

[3] Morton CA, Brown SB, Collins S, Ibbotson S, Jenkinson H, Kurwa H, Langmack K, McKenna K, Moseley H, Pearse AD, Stringer M, Taylor DK, Wong G, and Rhodes LE, "Guidelines for topical photodynamic therapy: report of a workshop of the British Photodermatology Group.", British Journal of Dermatology, 146, 4, 552-567, 2002.

[4] Bissonette R, Bergeron A, and Liu Y, "Large surface photodynamic therapy with aminolevulinic acid: treatment of actinic keratoses and beyond", Journal of Drugs in Dermatology, 3, 1 Suppl, S26-S31, 2004.

[5] Braathen LR, Szeimies RM, Basset-Seguin N, Bissonette R, Foley P, Pariser D, Roelandts R, Wennberg AM, and Morton CA, "Guidelines on the use of photodynamic therapy for nonmelanoma skin cancer: An international consensus.", Journal of the American Academy of Dermatology, 56, 1, 125-143, 2007.

[6] Morton CA, McKenna KE, and Rhodes LE, "Guidelines for topical photodynamic therapy: update", British Journal of Dermatology, 159, 6, 1245-1266, 2008.

[7] Wiegell SR, "Update on photodynamic treatment for actinic keratosis.", Current Problems in Dermatology, 46, 122-128, 2015.

[8] Kennedy JC, Pottier RH, and Pross DC, "Photodynamic therapy with endogenous protoporphyrin IX: basic principles and present clinical experience", Journal of Photochemistry and Photobiology B, 6, (1-2), 143-148, 1990.

[9] Peng Q, Warloe T, Berg K, Moan J, Kongdhaug M, Giercksky KE, and Nishimura G, "5Aminolevulinic acid-based photodynamic therapy. Clinical research and future challenges.", Cancer, 79, 12, 2282-2308, 1997. 
[10] Morton CA, Szeimies RM, Sidoroff A, and Braathen LR, "European guidelines for topical photodynamic therapy part 1: treatment delivery and current indications - actinic keratoses, Bowen's disease, basal cell carcinoma", Journal of the European Academy of Dermatology And Venereology: JEADV, 27, 5, 536-544, 2013.

[11] Piacquadio DJ, Chen DM, Farber HF, Fowler JF, Glazer SD, Goodman JJ, Hruza LL, Jeffes EWB, Ling MR, Phillips TJ, Rallis TM, Scher RK, Taylor CR, and Weinstein GD, "Photodynamic Therapy With Aminolevulinic Acid Topical Solution and Visible Blue Light in the Treatment of Multiple Actinic Keratoses of the Face and Scalp", Archives of Dermatology, 140, 1, 41-46, 2004.

[12] Morton C, Campbell S, Gupta G, Keohane S, Lear J, Zaki I, Walton S, Kerrouche N, Thomas G, and Soto P, "Intraindividual, right-left comparison of topical methyl aminolaevulinatephotodynamic therapy and cryotherapy in subjects with actinic keratoses: a multicentre, randomized controlled study", British Journal of Dermatology, 155, 5, 1029-1036, 2006.

[13] Morton CA, Whitehurst C, Moseley H, McColl JH, Moore JV, and Mackie RM, "Comparison of photodynamic therapy with cryotherapy in the treatment of Bowen's disease", British Journal of Dermatology, 135, 5, 766-771, 1996.

[14] Calzavara-Pinton PG, Venturini M, Sala R, Capezzera R, Parrinello G, Specchia C, and Zane $\mathrm{C}$, "Methylaminolaevulinate-based photodynamic therapy of Bowen's disease and squamous cell carcinoma", The Bristish Journal of Dermatology, 159, 1, 137-144, 2008.

[15] Basset-Seguin N, Ibbotson SH, Emtestam L, Tarstedt M, Morton C, Maroti M, CalzavaraPinton P, Varma S, Roelandts R, and Wolf P, "Topical methyl aminolaevulinate photodynamic therapy versus cryotherapy for superficial basal cell carcinoma: a 5 year randomized trial", European Journal of Dermatology, 18, 5, 547-553, 2008.

[16] Szeimies RM, Ibbotson S, Murrell DF, Rubel D, Frambach Y, De Berker D, Dummer R, Kerrouche N, and Villemagne $\mathrm{H}$, "A clinical study comparing methyl aminolevulinate photodynamic therapy and surgery in small superficial basal cell carcinoma $(8-20 \mathrm{~mm})$, with a 12-month follow-up", Journal of the European Academy of Dermatology And Venereology: JEADV, 22, 11, 1302-1311, 2008.

[17] Tyrrell J, Campbell SM, and Curnow A, "The effect of air cooling pain relief on protoporphyrin IX photobleaching and clinical efficacy during dermatological photodynamic therapy", Journal of Photochemistry and Photobiology, 103, 1, 1-7, 2011.

[18] Stangeland KZ and Kroon S, "Cold air analgesia as pain reduction during photodynamic therapy of actinic keratoses", Journal of the European Academy of Dermatology And Venereology: JEADV, 26, 7, 849-854, 2012.

[19] Arits AH, Van de Weert MM, Nelemans PJ, and Kelleners-Smeets NW, "Pain during topical photodynamic therapy: uncomfortable and unpredictable", Journal of the European Academy of Dermatology And Venereology: JEADV, 24, 12, 1452-1457, 2010.

[20] Apalla Z, Sotiriou E, Panagiotidou D, Lefaki I, Goussi C, and Ioannides D, "The impact of different fluence rates on pain and clinical outcome in patients with actinic keratoses treated with photodynamic therapy.", Photodermatology, Photoimmunology \& Photomedicine, 27, 4, 181-185, 2011.

[21] Tschen EH, Wong DS, Pariser DM, Dunlap FE, Houlihan A, and Ferdon MB, "Photodynamic therapy using aminolaevulinic acid for patients with nonhyperkeratotic actinic keratoses of the 
face and scalp: phase IV multicentre clinical trial with 12-month follow up.", British Journal of Dermatology, 155, 6, 1262-1269, 2006.

[22] Wiegell SR, Hædersdal M, Philipsen PA, Eriksen P, Enk CD, and Wulf HC, "Continuous activation of PpIX by daylight is as effective as and less painful than conventional photodynamic therapy for actinic keratoses; a randomized, controlled, single-blinded study", The Bristish Journal of Dermatology, 158, 4, 740-746, 2008.

[23] Valentine RM, Brown CT, Moseley H, Ibbotson S, and Wood K, "Monte Carlo modeling of in vivo protoporphyrin IX fluorescence and singlet oxygen production during photodynamic therapy for patients presenting with superficial basal cell carcinomas", Journal of Biomedical Optics, 16, 4, 048002- 2011.

[24] Liu B, Farrell TJ, and Patterson MS, "A dynamic model for ALA-PDT of skin: simulation of temporal and spatial distributions of ground-state oxygen, photosensitizer and singlet oxygen", Physics in Medicine and Biology, 55, 5912-5932, 2010.

[25] Liu B, Farrell TJ, and Patterson MS, "Comparison of noninvasive photodynamic therapy dosimetry methods using a dynamic model of ALA-PDT of human skin", Physics in Medicine and Biology, 57, 825-841, 2012.

[26] Wang Y, Gu Y, Zuo Z, and Huang N, "Choosing optimal wavelength for photodynamic therapy of port wine stains by mathematic simulation", Journal of Biomedical Optics, 16, 9, 2011.

[27] Hennig G, Stepp H, and Johansson A, "Photobleaching-based method to individualize irradiation time during interstitial 5-aminolevulinic acid photodynamic therapy", Photodiagnosis and Photodynamic Therapy, 8, 3, 275-281, 2011.

[28] Farrell TJ, Hawkes RP, Patterson MS, and Wilson BC, "Modeling of photosensitizer fluorescence emission and photobleaching for photodynamic therapy dosimetry", Applied Optics, 37, 31, 7168-7183, 1998.

[29] Carp SA, Prahl SA, and Venugopalan V, "Radiative transport in the delta-P1 approximation: accuracy of fluence rate and optical penetration depth predictions in turbid semi-infinite media", Journal of Biomedical Optics, 9, 3, 632-647, 2004.

[30] Moseley H, "Light distribution and calibration of commercial PDT LED arrays", Photochemical \& Photobiological Sciences, 4, 911-914, 2005.

[31] Tyrrell J, Campbell SM, and Curnow A, "Protoporphyrin IX photobleaching during the light irradiation phase of standard dermatological methyl-aminolevulinate photodynamic therapy", Photodiagnosis and Photodynamic Therapy, 7, 232-238, 2010.

[32] DUSA Pharmaceuticals, "Levulan Kerastick topical solution 20\%", Package insert http://www.dusapharma.com, 7-6-2009.

[33] Warren CB, Lohser S, and Wen LC, "Noninvasive fluorescence monitoring of protoporphyrin IX production and clinical outcomes in actinic keratoses following short-contact application of 5-aminolevulinate", Journal of Biomedical Optics, 15, 5, 051607- 2010.

[34] "http://www.pveducation.org/pvcdrom/appendicies/standard-solar-spectra", Internet, 2014. 
[35] Campbell CL, Wood K, Brown CTA, Valentine RM, and Moseley H, "Mathematical modeling of daylight activated photodynamic therapy for continuous accumulation of PpIX", Laser Europe Conference 2014, 2014.

[36] Dysart JS, Singh G, and Patterson MS, "Calculation of singlet oxygen dose from photosensitizer fluorescence and photobleaching during mTHPC photodynamic therapy of MLL cells", Photochemistry and Photobiology, 81, 196-205, 2005.

[37] Salomatina E, Jiang B, Novak J, and Yaroslavsky AN, "Optical properties of normal and cancerous human skin in the visible and near-infrared spectral range", Journal of Biomedical Optics, 11, 6, 064026- 2006.

[38] Garcia-Uribe A, Zou J, Duvic M, Cho-Vega JH, Prieto VG, and Wang LV, "In Vivo diagnosis of melanoma and nonmelanoma skin cancer using oblique incidence diffuse reflectance spectrometry", Cancer Research, 72, 11, 2738-2745, 2012.

[39] Star WM, Aalders MCG, Sac A, and Sterenborg HJCM, "Quantitative Model Calculation of the Time-dependent Protoporphyrin IX Concentration in Normal Human Epidermis After Delivery of ALA by Passive Topical Application or lontophoresis", Photochemistry and Photobiology, 2002, 75, 4-424, 2002.

[40] Angell-Petersen E, Sorensen R, Warloe T, Soler AM, Moan J, Peng Q, and Giercksky KE, "Porphyrin Formation in Actinic Keratosis and Basal Cell Carcinoma after Topical Application of Methyl 5-Aminolevulinate", Journal of Investigative Dermatology, 126, 265$271,2006$.

[41] Bonda CA and Hu S, "Tricyclic energy quencher compounds for reducing singlet oxygen generation", Patent WO2014025370 A1, 2014.

[42] Wilkinson F, Helman WP, and Ross AB, "Quantum Yields for the Photosensitized Formation of the Lowest Electronically Excited Singlet State of Molecular Oxygen in Solution", Journal of Physical and Chemical Reference Data, 22, 113-262, 1993.

[43] Fernandez JM, Bilgin MD, and Grossweiner LI, "Singlet oxygen generation by photodynamic agents", Journal of Photochemistry and Photobiology B, 37, 1, 131-140, 1997.

[44] Smits T and Moor ACE, "New aspects in photodynamic therapy of actinic keratoses", Journal of Photochemistry and Photobiology, 96, 3, 159-169, 2005.

[45] Christiansen K, Bjerring P, and Troilius A, "5-ALA for Photodynamic PhotorejuvenationOptimization of Treatment Regime Based on Normal-Skin Fluorescence Measurements", Lasers in Surgery and Medicine, 39, 302-310, 2007.

[46] Natarajan P and Raja C, "Studies on interpolymer self-organisation behaviour of protoporphyrin IX bound poly(carboxylic acid)s with complimentary polymers by means of fluorescence techniques", European Polymer Journal, 40, 2291-2303, 2004.

[47] Szeimies RM, Matheson RT, Davis SA, Bhatia AC, Frambach Y, Klövekorn W, Fesq H, Berking C, Reifenberger J, and Thaçi D, "Topical methyl aminolevulinate photodynamic therapy using red light-emitting diode light for multiple actinic keratoses: a randomized study", Dermatologic Surgery, 35, 4, 586-592, 2009. 
[48] Wan MT and Lin JY, "Current evidence and applications of photodynamic therapy in dermatology", Clinical, cosmetic and investigational dermatology, 21, 7, 145-163, 2014.

[49] Woodhams JH, Macrobert AJ, and Bown SG, "The role of oxygen monitoring during photodynamic therapy and its potential for treatment dosimetry", Photochemical \& Photobiological Sciences, 6, 12, 1246-1256, 2007.

[50] Langmack K, Mehta R, Twyman P, and Norris P, "Topical photodynamic therapy at low fluence rates--theory and practice.", Journal of Photochemistry and Photobiology B, 60, 1, 37 43, 2001.

[51] Stücker M, Struk A, Altmeyer P, Herde M, Baumgärtl H, and Lübbers DW, "The cutaneous uptake of atmospheric oxygen contributes significantly to the oxygen supply of human dermis and epidermis", The Journal of Physiology, 538, 3, 985-994, 2002. 